\title{
Hematological Profile in Patients with H1N1 - A Retrospective Analysis of 158 Cases
}

\author{
Dr. Yegumuthu $\mathrm{K}^{1}$, Dr. Poorana Priya $\mathrm{P}^{2^{*}}$, Dr. Subbiah $\mathrm{M}^{3}$
}

${ }^{1}$ Associate Professor, Department of Pathology, Velammal Medical College Hospital, Madurai Tamil Nadu India

${ }^{2}$ Assistant Professor, Department Of Pathology, Velammal Medical College Hospital, Madurai Tamil Nadu India

${ }^{3}$ Associate Professor, Department Of Orthopaedics, Velammal Medical College Hospital, Madurai Tamil Nadu India

DOI: $10.36347 /$ sjams.2020.v08i05.029

| Received: 21.04.2020 | Accepted: 09.05.2020 | Published: 22.05.2020

*Corresponding author: Dr. Poorana Priya P

Abstract

Original Research Article

H1N1 [Swine flu], a novel strain of Influenza A virus, has contributed to significant morbidity and mortality. The first case in India was identified in May 2009. Several outbreaks have occurred since then, yet very few studies on complete blood analysis of H1N1Influenza have been reported. We conducted a retrospective study in 158 adult patients for whom H1N1 Influenza A virus was detected by RT - PCR method. Complete blood count reports of all the RT PCR positive patients were collected from the hospital information system and the laboratory data were retrospectively assessed and analysed. RT - PCR is considered as the gold standard for the diagnosis of H1N1 Influenza. Although RT PCR is a sensitive test, results are often delayed leading to difficulties in disease management. Rapid diagnosis of H1N1 Influenza is important for early initiation of anti viral therapy. Hence we analyzed the haematological parameters of H1N1 patients to identify a potential marker for H1N1 infection in order to help the clinicians to plan the treatment protocol for patients presenting with Influenza like symptoms while awaiting the PCR / culture reports. We could demonstrate that, lymphopenia and eosinopenia in the presence of clinical parameters may be helpful in identifying the cases of H1N1 while more sensitive laboratory test results are awaited.

Keywords: H1N1, Influenza, Lymphopenia, Eosinopenia.

Copyright @ 2020: This is an open-access article distributed under the terms of the Creative Commons Attribution license which permits unrestricted use, distribution, and reproduction in any medium for non-commercial use (NonCommercial, or CC-BY-NC) provided the original author and source are credited.

\section{INTRODUCTION}

H1N1 Influenza A viruses are associated with recurrent epidemics and occasional global pandemics [1]. In India, the first confirmed case of H1N1 swine flu was identified in May 2009 but thereafter, a huge number of cases have been reported [2]. Since then $\mathrm{H} 1 \mathrm{~N} 1$ swine flu has contributed to significant morbidity and mortality [3].

Influenza a viruses belong to Orthomyxoviridae family. They are classified into 16 HA subtypes [H1 to H16] and 9 NA [N1 to N9] subtypes based on the antigenicity of their hemagglutinin [HA] and neuraminidase [NA] molecules [4]. H1N1 is a novel strain of Influenza A Virus which has evolved by genetic re assortment. [5] Influenza is a common virus which causes a spectrum of respiratory infection ranging in severity from mild flu to life threatening pneumonia, bronchitis, acute respiratory distress syndrome and even death[2,3]. Hence, prevention, early laboratory diagnosis and treatment of the disease especially the severely ill cases are of utmost importance. Reverse transcriptase polymerase chain reaction and throat swab culture are the preferred diagnostic tests for H1N1 Influenza. However, both these tests are time consuming which can result in significant delay in confirmation of suspected cases and their subsequent isolation [5]. The present study aims at analysing the haematological profile in patients with swine flu and identifying a reliable laboratory parameter which can serve as a marker for $\mathrm{H} 1 \mathrm{~N} 1$ infection in patients presenting with Influenza like symptoms while waiting for throat swab culture and RT PCR Reports.

\section{Materials And Methods}

This retrospective study was conducted in our tertiary hospital. Laboratory data of 171 confirmed cases of swine flu in the year 2018 were retrieved from the records. Complete blood counts were obtained on the day of their admission from all the adult patients [aged more than 18 years] in whom Influenza a H1N1 was detected by RT PCR method. Complete blood counts were performed by automated analyzer Beckman coulter LH 750. Laboratory parameters were recorded and retrospectively assessed. Pediatric cases and patients with haematological malignancies were excluded from the study. 


\section{RESULTS AND DiSCUSSION}

Table -1: Age and sex distribution of Influenza a H1N1 patients

\begin{tabular}{|l|l|l|l|l|}
\hline Age in years & Male & Female & Total & Percentage \\
\hline $18-30$ & 9 & 10 & 19 & $12.02 \%$ \\
\hline $31-40$ & 12 & 6 & 18 & $11.39 \%$ \\
\hline $41-50$ & 16 & 9 & 25 & $15.82 \%$ \\
\hline $51-60$ & 18 & 27 & 45 & $28.48 \%$ \\
\hline $61-70$ & 14 & 14 & 28 & $17.72 \%$ \\
\hline $71-80$ & 10 & 8 & 18 & $11.39 \%$ \\
\hline $81-90$ & 3 & 2 & 5 & $3.16 \%$ \\
\hline Total & 82 & 76 & 158 & $100 \%$ \\
\hline
\end{tabular}

Table-2: Summarizes the Haematological findings of study subjects

\begin{tabular}{|l|l|}
\hline Variable & Mean [ $\mathrm{n}=158$ ] \\
\hline RBC count [ millions / cu.mm] & $4.37 \pm 0.76$ \\
\hline Hemoglobin [ gm / dl ] & $12.23 \pm 2.22$ \\
\hline Leucocyte count [ cells / cu.mm ] & $10,120 \pm 6984$ \\
\hline Platelet count [ 1000 / cu.mm ] & $2.25 \pm 0.76$ \\
\hline Neutrophil lymphocyte ratio & $9.16 \pm 9.99$ \\
\hline
\end{tabular}

Table-3: Summarizes the blood cell analysis in H1N1 patients

\begin{tabular}{|l|l|l|l|}
\hline \multirow{3}{*}{ RBC COUNT } & Decreased & $\mathbf{7 8}$ & $\mathbf{4 9 . 3 6 \%}$ \\
\cline { 2 - 4 } & Normal & 77 & $48.73 \%$ \\
\cline { 2 - 4 } & Increased & 3 & $1.89 \%$ \\
\hline \multirow{3}{*}{ WBC COUNT } & Decreased & 17 & $10.7 \%$ \\
\cline { 2 - 4 } & Normal & 96 & $60.7 \%$ \\
\cline { 2 - 4 } & Increased & 45 & $28.4 \%$ \\
\hline PLATELET COUNT & Decreased & 24 & $15.18 \%$ \\
\cline { 2 - 4 } & Normal & 132 & $83.5 \%$ \\
\cline { 2 - 4 } & Increased & 2 & $1.26 \%$ \\
\hline
\end{tabular}

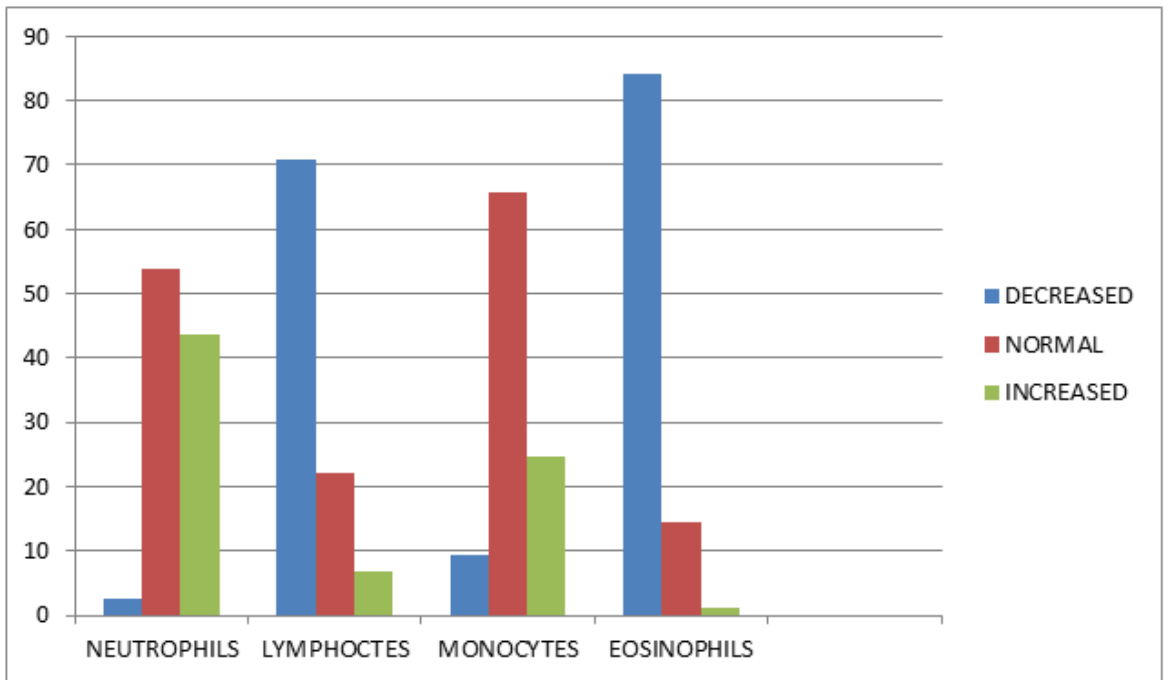

Fig-1: Comparison of Differential Leucocyte Count in patients with H1N1

This study reveals the hematological profile of patients with H1N1 Influenza. Our study showed that while the higher incidence of swine flu occurred between the ages of $51-60$ years [28.48 \%], the mean age of the patients was 52.3 years with a male to female ratio of 1.08: 1.0. In the study conducted by George Merekoulias et al. [6], H1N1 was found to be more common in younger individuals. The difference in age group may be attributed to exclusion of pediatric cases in our study or a change in the demographic trend with increasing number of cases over the years. A large scale, community based, prospective study [7] showed that $\mathrm{H} 1 \mathrm{~N} 1$ preferentially affected young children during the pandemic phase with a shift to adults in the post 
pandemic phase. The study also suggested that the increased incidence of infection in children led to acquired immunity thereby decreasing the susceptibility to infection during the post pandemic phase.

Our results showed that most of the patients had a normal or slightly decreased haemoglobin levels. The total WBC count varied from 1800 to 48800cells/cu.mm. Studies carried out by Wang et al. [3] demonstrated leukopenia in $90 \%$ of cases whereas that of George Merekoulias et al. [6] showed a normal WBC count in $90 \%$ of cases. Contrary to their findings, we noted that leukopenia was observed only in $10.7 \%$ whereas normal WBC count was observed in $60.7 \%$ and leukocytosis in $28.45 \%$ of patients.

In majority of the cases, the differential count showed neutrophilic predominance with a decrease in the number of lymphocytes. Studies by Dhanoa et al. [8] have shown that bacterial co infection was common in patients with H1N1 Influenza especially in the older age group. According to their study patients with bacterial co-infection demonstrated higher rates of neutrophilia and leukocytosis. Our study demonstrated neutrophilia in $43.6 \%$ and leukocytosis in $28.45 \%$ of cases.

Lymphopenia was a consistent finding in previous studies $[6,9,10]$ and it was observed in our study as well. Relative lymphopenia (less than or equal to $20 \%$ of $\mathrm{WBC}$ ) was present in 112 of 158 patients. As shown in studies by Wang et al. [3], the ratio of lymphocytes are often increased in common respiratory illnesses such as those caused by Rhinovirus and Respiratory syncytial virus? Hence, Lymphopenia can be considered as a screening parameter for $\mathrm{H} 1 \mathrm{~N} 1$, in patients presenting with respiratory illness.

The Neutrophil Lymphocyte Ratio [NLR] was calculated as neutrophils / lymphocytes. The mean NLR in our study was $9.16 \pm 9.99$ which was comparable to studies by quinzhen et al. [11] which showed a mean NLR of $8.87 \pm 9.85$ and a much higher level $18.93 \pm$ 38.44 was seen in bacterial infections.

Our study demonstrated eosinopenia (less than $1 \%$ of WBC count) in $133 / 158$ patients. $123 / 158$ patients had an eosinophil count of less than or equal to $0.5 \%$. In fact, the eosinophil count was zero in $63 / 158$ patients. Flick et al. [12] proposed that combined evaluation of clinical and laboratory parameters were clearly superior when compared to clinical criteria alone. According to the study, simple laboratory parameters like leukopenia, eosinopenia and decreased $\mathrm{C}$ reactive protein levels were significant predictors of H1N1 Influenza.

In our experience, we could demonstrate lymphopenia in $70.8 \%$ and eosinopenia in $84.1 \%$ of cases. Comparable to our study, the results obtained from a study by Shen et al. [13] demonstrated lymphopenia in $96.4 \%$ and eosinopenia in $50 \%$ of their cases.

Thrombocytopenia was seen in 24 of 158 adults. Thrombocytopenia often accompanies influenza A virus infection and varied mechanisms like decreased production, splenic sequestration and direct or indirect platelet activation which promotes platelet - leukocyte aggregation have been implicated as possible causal factors for thrombocytopenia [14]. Studies have also shown that thrombocytopenia is a significant risk factor for mortality in patients with influenza a virus infection [15].

\section{CONCLUSION}

Early diagnosis of $\mathrm{H} 1 \mathrm{~N} 1$ Influenza is mandatory to begin preventive measures and commence antiviral treatment. Hematological profile is a simple, effective and a routine investigation done in all admitted patients. In our experience, lymphopenia was a constant finding in H1N1 Influenza, when compared to leukopenia or thrombocytopenia. Lymphopenia and eosinopenia along with other clinical parameters may be valuable aids in the diagnosis of H1N1 Influenza. Hence routine screening for haematological profile in patients presenting with influenza like illness is advocated to initiate early management strategies and decrease the morbidity related to it.

\section{REFERENCES}

1. Neumann G, Noda T, Kawaoka Y. Emergence and pandemic potential of swine-origin H1N1 influenza virus. Nature. 2009 Jun;459(7249):931-9.

2. Kshatriya RM, Khara NV, Ganjiwale J, Lote SD, Patel SN, Paliwal RP. Lessons learnt from the Indian H1N1 (swine flu) epidemic: Predictors of outcome based on epidemiological and clinical profile. Journal of family medicine and primary care. 2018 Nov;7(6):1506.

3. Wang N, Liu X, Zhang Y, Xie Y, Zhao W. Hematologic markers of influenza A H1N1 for early laboratory diagnosis and treatment assessment. Laboratory Medicine. 2011 Oct 1;42(10):607-11.

4. Taubenberger JK, Morens DM. The pathology of influenza virus infections. Annu. Rev. Pathol. Mech. Dis.. 2008 Feb 28;3:499-522.

5. Indavarapu A, Akinapelli A. Neutrophils to lymphocyte ratio as a screening tool for swine influenza. The Indian journal of medical research. 2011 Sep;134(3):389.

6. Merekoulias G, Alexopoulos EC, Belezos T, Panagiotopoulou E, Jelastopulu E. Lymphocyte to monocyte ratio as a screening tool for influenza. PLoS currents. 2010 Mar 30;2.

7. Broor S, Sullender W, Fowler K, Gupta V, Widdowson MA, Krishnan A, Lal RB. Demographic shift of influenza A (H1N1) pdm09 
during and after pandemic, rural India. Emerging infectious diseases. 2012 Sep;18(9):1472.

8. Dhanoa A, Fang NC, Hassan SS, Kaniappan P, Rajasekaram G. Epidemiology and clinical characteristics of hospitalized patients with pandemic influenza A (H1N1) 2009 infections: the effects of bacterial coinfection. Virology journal. 2011 Dec 1;8(1):501.

9. Cunha BA, Pherez FM, Schoch P. Diagnostic importance of relative lymphopenia as a marker of swine influenza (H1N1) in adults. Clinical infectious diseases. 2009 Nov 15;49(9):1454-6.

10. Cheng Y, Zhao H, Song P, Zhang Z, Chen J, Zhou YH. Dynamic changes of lymphocyte counts in adult patients with severe pandemic H1N1 influenza A. Journal of infection and public health. 2019 Nov 1;12(6):878-83.

11. Han Q, Wen X, Wang L, Han X, Shen Y, Cao J, Peng Q, Xu J, Zhao L, He J, Yuan H. Role of hematological parameters in the diagnosis of influenza virus infection in patients with respiratory tract infection symptoms. Journal of Clinical Laboratory Analysis. 2020 Jan 4:e23191.
12. Flick H, Drescher M, Prattes J, Tovilo K, Kessler HH, Vander K, Seeber K, Palfner M, Raggam RB, Avian A, Krause R. Predictors of H1N1 influenza in the emergency department: proposition for a modified H1N1 case definition. Clinical Microbiology and Infection. 2014 Feb 1;20(2):O105-8.

13. Shen H, Li B, Bai B, Hou J, Xu Z, Zhao M, Mao P, Liu A, Li J, Guo T, Xu J. Laboratory features throughout the disease course of influenza A (H1N1) virus infection. Clinical laboratory. 2013;59(3-4):337-42.

14. Boilard E, Paré G, Rousseau M, Cloutier N, Dubuc I, Lévesque T, Borgeat P, Flamand L. Influenza virus $\mathrm{H} 1 \mathrm{~N} 1$ activates platelets through FcyRIIA signaling and thrombin generation. Blood, The Journal of the American Society of Hematology. 2014 May 1;123(18):2854-63.

15. Lopez-Delgado JC, Rovira A, Esteve F, Rico N, Mañez Mendiluce R, Ballús Noguera J, Berrade J. Thrombocytopenia as a mortality risk factor in acute respiratory failure in $\mathrm{H} 1 \mathrm{~N} 1$ influenza. Swiss medical weekly. 2013 Apr 18;143(1516). 